\title{
BISTATIC SYSTEM FOR RADAR SENSING OF SOIL MOISTURE
}

\author{
Anatoliy Bazhenov ${ }^{1}$, Konstantin Sagdeev ${ }^{1}$, Dmitry Goncharov ${ }^{1}$, Natalia Grivennaya ${ }^{2}$ \\ ${ }^{1}$ North-Caucasian Federal University, Russia; ${ }^{2}$ Stavropol State Pedagogical Institute, Russia \\ a_bazenov61@rambler.ru,skm63@yandex.ru,dggoncharov@ncfu.ru, katrinastenton@yandex.ru
}

\begin{abstract}
The article is devoted to the development of a method for determining the moisture content of the upper layers of agricultural lands on the basis of mathematical modeling of a radar signal reflected from the soil. For the radar study of the moisture content of agricultural lands, it is proposed to use remote sensing using relatively inexpensive unmanned aerial vehicles (UAVs) of a multi-rotor type (a bistatic system consisting of a radar transmitter and a radar receiver placed on two UAVs). At the same time, it is necessary to ensure a high probability of correct determination of soil moisture to a depth exceeding the groundwater level. In remote sensing, the reflection coefficient from the air-soil interface depends on the dielectric properties of the top soil layer and the polarization of the sounding signal. When modeling the reflected signal, it is advisable to represent the earth's surface as a flat-layered structure, the upper part of which will be represented by vegetation and heterogeneities followed by the area with a relatively constant dielectric constant value to the groundwater level and then the area with a linearly increasing conductivity. Studies have shown that the Brewster effect in soils manifests itself when the probe signal is perpendicularly polarized. When the soil surface is irradiated with a perpendicularly polarized radar signal at Brewster's angle, the required penetration depth of the probing signal is ensured, at which the reflected signal will contain enough information about the percentage of moisture in the upper soil layers. The information contained in changes in the amplitude and phase of the reflected radio signal is related to the structure and dielectric properties of the upper soil layers. On the basis of this information, rational agro-technical measures can be justified, both operational (watering, fertilization) and long-term (reclamation, drainage).
\end{abstract}

Keywords: GRP sounding, soil moisture, UAV, bistatic system, mathematical modeling.

\section{Introduction}

Radar sounding is based on the extraction of information from the reflected signal; the information consists of changes in the amplitude and phase associated with the structure and dielectric properties of the topsoil [1-2].

Depending on the area of application and the tasks to be solved, different frequencies, polarizations, and structures of the probing signal are used [3;4]. Depending on the location of the radar equipment (georadar, GPR), ground-based, satellite, and aviation (including unmanned) radar sounding systems are distinguished [5].

The use of ground-based GPRs is significantly limited due to the influence of the sounding signal source on the antenna system and some other reasons.

Therefore, for the radar studies of the agricultural land moisture content, it is advisable to use remote sensing using relatively inexpensive multi-rotor unmanned aerial vehicles (UAVs) of the multi-rotor type. At the same time, it is necessary to ensure a high probability of correct determination of soil moisture to a depth exceeding the groundwater level, which in the conditions of the Russian Federation is $2.5-4 \mathrm{~m}$.

In remote sensing, the reflection coefficient from the air-ground interface depends on the dielectric properties of the topsoil layer and the polarization of the sounding signal [6-7]. As shown in Fig. 1, with the vertical polarization of the radar signal, there is an angle of incidence (Brewster's angle), at which the reflection coefficient from the dielectric boundary takes a zero value [8]. Thus, it may be assumed that when irradiating the surface at Brewster's angle, the required penetration depth of the probing signal will be provided at which the reflected signal will contain sufficient information about the moisture percentage in the upper soil layers.

The research aim is to develop a method for determining the moisture content of the upper layers of agricultural lands based on the processing of the reflected radar signal using a bistatic system consisting of a radar transmitter and a radar receiver placed on two UAVs.

\section{Materials and methods}

From the electrodynamic point of view, soils are a heterogeneous dispersed medium consisting of soil particles of several types with different sizes and mineral compositions. 


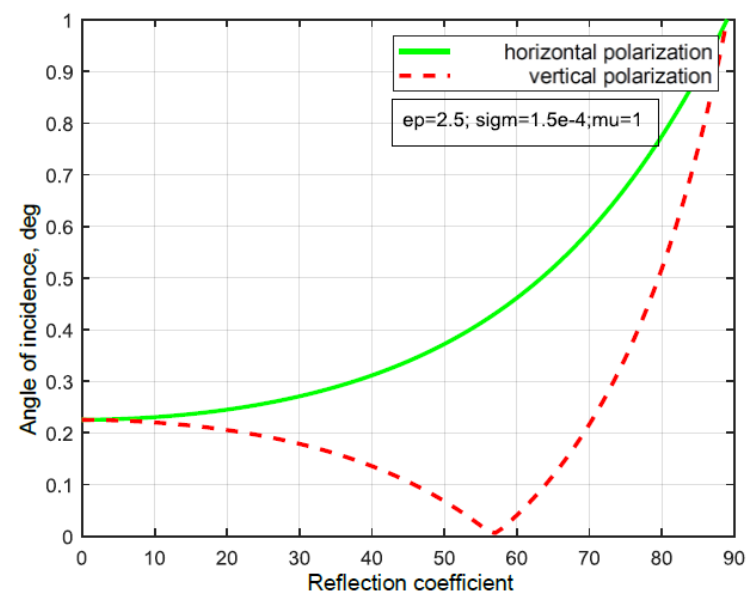

Fig. 1. Dependence of the reflection coefficient on the incidence angle and polarization of an electromagnetic wave

The influence of soil moisture on its dielectric properties has been studied in a large number of papers, among which one can distinguish [9-10]. At the same time, analytical modeling of the soils' dielectric properties seems to be a difficult task. As a rule, either empirical and semi-empirical models or electrostatic models of mixtures are used for these purposes [11-12].

To simulate the reflected signal, the Earth's surface should be represented as a flat-layered structure, upper part with vegetation and inhomogeneities followed by a layer with a relatively constant dielectric permeability value up to the groundwater level and then a section with a linearly increasing conductivity [13]. Let us consider the formation of the reflected signal, when using a type (4) continuous monochromatic radio signal when irradiating the Earth's surface along the normal:

$$
u_{0}(t)=U_{0} \exp (-j \omega t)
$$

where $U_{0}$-amplitude;

$\omega=2 \pi f$ - probing signal cyclic frequency.

The received reflected signal can be represented as a sum of signals generated at the boundaries of separate $i$-th layers:

$$
U_{\text {refl }}(t)=\sum_{i=1}^{N} u_{i}(t) .
$$

For the first boundary at the transition from air to soil, the reflected signal can be written as follows:

$$
u_{1}(t)=U_{0} \cdot K_{d} \cdot R_{01} \cdot \exp (-j \omega(t+2 H / c),
$$

where $K_{d}$-coefficient that takes into account the nature of reflection (diffuse, specular or mixed) from inhomogeneities and vegetation on the soil surface;

$R_{01}$ - reflection coefficient which determines the fraction of energy reflected from the airsoil boundary;

$H$ - height at which radar sounding is carried out;

$c$ - phase velocity of an electromagnetic wave in the air, taken equal to the speed of light in vacuum.

The UAV flight altitude is selected to ensure that the air-ground boundary is located in the far zone of the radiating antenna.

At the next layer boundary, the expression for the probing signal will take the following form:

$$
u(t)=U_{0} \cdot K_{d} \cdot T_{01} \cdot \exp \left(-\alpha_{1} \cdot \Delta z_{1}\right) \cdot \exp (-j \omega t),
$$

where $T_{01}-$ refractive index at the air-soil interface;

$\alpha_{1}$-attenuation coefficient, determined by the electrical properties of the topsoil;

$\Delta z_{1}$ - width of the topsoil.

In general, the signal reflected from the boundary of $i$ and $i+1$ layers can be written as an expression: 


$$
u_{i+1}(t)=U_{i} \cdot R_{i i+1} \cdot \exp (-j \omega(t+2 H / c)) \cdot \exp \left(4 \pi\left(\Delta z_{1}+\Delta z_{2}+\ldots+\Delta z_{i}\right) / \lambda\right),
$$

where $\lambda$-wavelength of the probing radiation.

Depending on the polarization of the electromagnetic wave, determined by the position of the electric field vector in space during propagation, the formulas for calculating the reflection and refraction coefficients differ.

The reflection coefficient for vertical polarization is determined by the expression

$$
\dot{R}_{v}=\frac{Z_{c 1} \cos \phi-Z_{c 2} \cos \varphi}{Z_{c 1} \cos \phi+Z_{c 2} \cos \varphi}
$$

where $\quad \dot{Z}_{c}=\sqrt{\mu_{0} \mu_{r} / \varepsilon_{0} \varepsilon_{r}-j(\sigma / \omega)}-$ characteristic (wave) resistance of the medium;

Index 1 - corresponds to the medium in which the incident and reflected waves are located; index 2 - corresponds to the medium in which the refracted wave is located;

$\mu_{0}=4 \pi \cdot 10^{-7}$ is the vacuum magnetic permeability;

$\mu_{\mathrm{r}}$ is the relative magnetic permeability;

$\varepsilon_{0}=(1 / 36 \pi) \cdot 10^{-9}$ is the permittivity of vacuum;

$\varepsilon_{\mathrm{r}}$ is the relative dielectric permittivity;

$\sigma$ is the specific conductivity of the medium;

$\varphi$ - angle of incidence of the electromagnetic wave;

$\phi-$ refraction angle.

The refractive index for vertical polarization is determined by the expression

$$
\dot{T}_{v}=\frac{2 Z_{c 2} \cos \phi}{Z_{c 1} \cos \phi+Z_{c 2} \cos \varphi} .
$$

In the case of horizontal polarization, the reflection and refraction coefficients are determined by the expressions (8) and (9), respectively.

$$
\begin{aligned}
\dot{R}_{g} & =\frac{Z_{c 2} \cos \phi-Z_{c 1} \cos \varphi}{Z_{c 2} \cos \phi+Z_{c 1} \cos \varphi}, \\
\dot{T}_{g} & =\frac{2 Z_{c 2} \cos \phi}{Z_{c 2} \cos \phi+Z_{c 1} \cos \varphi} .
\end{aligned}
$$

As follows from Fig. 1, the Brewster effect manifests itself in the case of vertical polarization of the probe signal. Therefore, we will choose this type of polarization for the GPR operation.

After analyzing the obtained simulation results (see Fig. 4), the following conclusions can be drawn.

1. When the earth's surface is irradiated by a single-position geolocator with a continuous signal at angles close to the normal, the signal reflected from the air-soil boundary has the greatest amplitude.

2. Reflection picking from the inner layers can be carried out by the phase method.

3. To eliminate the ambiguities of the phase method, it is necessary to choose the wavelength of the probing signal that exceeds the desired depth of penetration into the soil (the distance from the surface to the assumed groundwater level).

Let us consider the process of soil moisture research using a bistatic system with two UAVs and irradiating the earth's surface at angles close to Brewster's angle value (Fig. 2).

When performing calculations according to expressions (4), (5) for each transition from one layer to another, it is necessary to take into account that the angle of incidence for the next layer will be equal to the angle of refraction of the previous one. The calculation of the angle of refraction value is performed on the basis of Snell's second law (Fig. 3):

$$
\frac{\sin \varphi}{\sin \phi}=\frac{n_{2}}{n_{1}}
$$

where $n=\frac{n_{1}}{n_{2}}=\sqrt{\mu_{r} \cdot\left(\varepsilon_{r}-j\left(\sigma / \varepsilon_{0} \omega\right)\right.}$ is the relative refractive index at

$$
\mu_{r}=1, n=\sqrt{\varepsilon_{r}-60 \lambda \sigma} \text {. }
$$




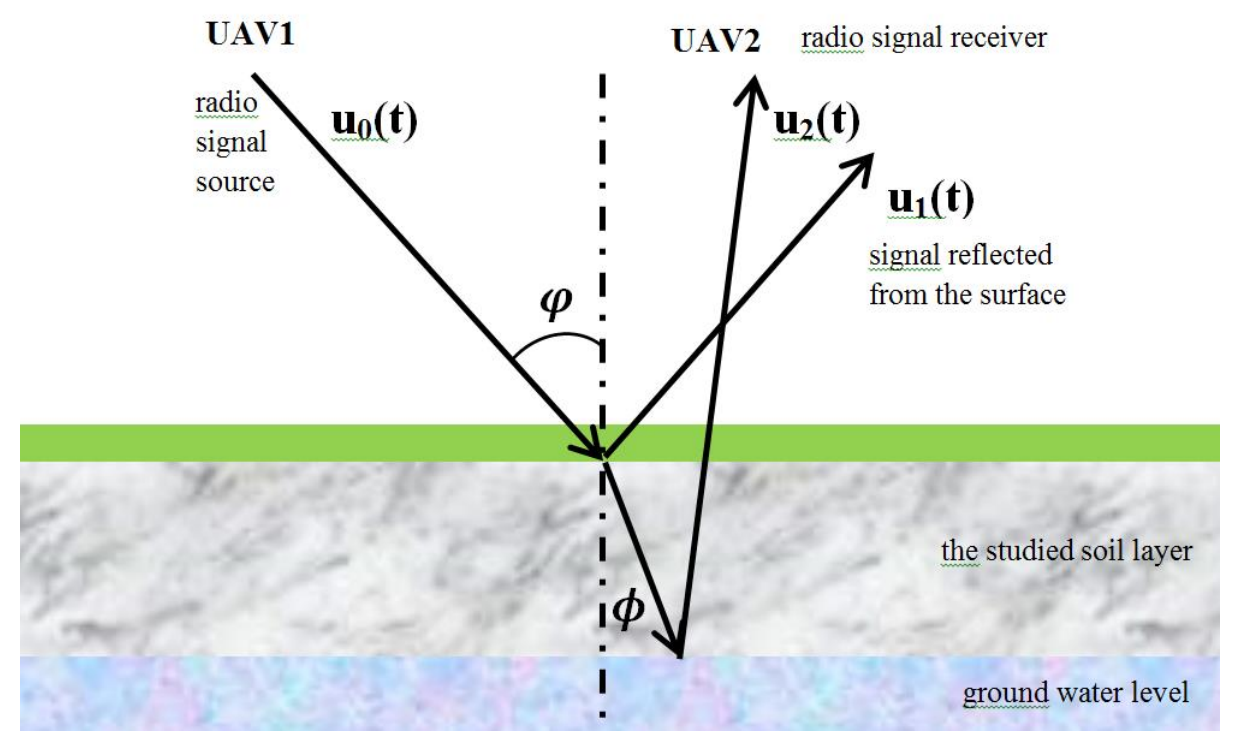

Fig. 2. Using a bistatic radar system to study soil moisture

a)

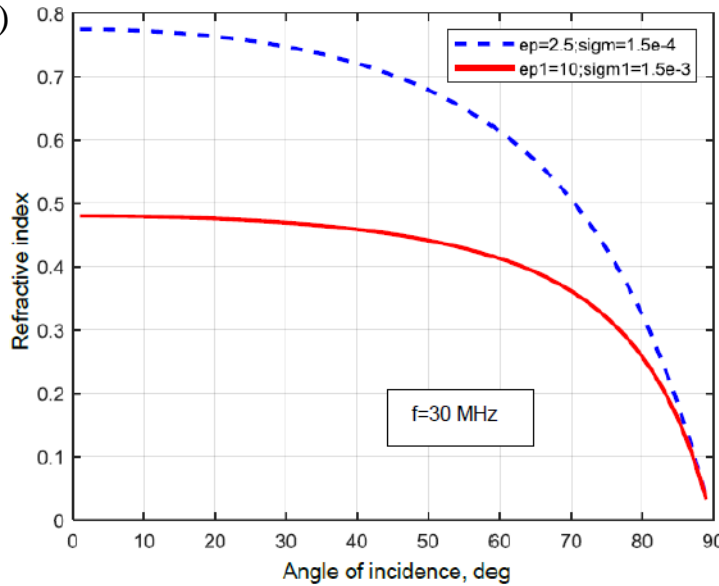

b)

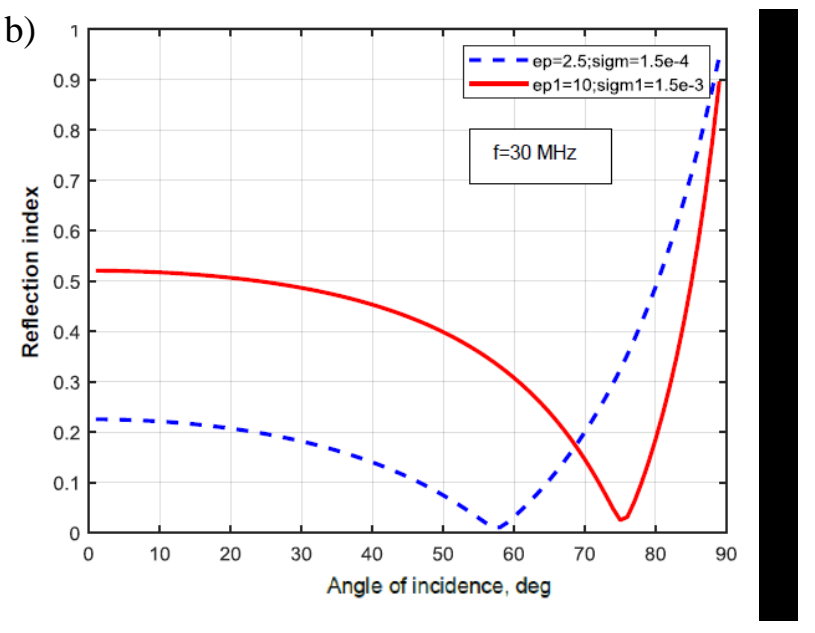

Fig. 3. Dependence of the reflection (a) and refraction (b) coefficients on the angle of incidence and the parameters of the upper and lower soil layers of different degrees of humidity

The simulation results shown in Fig. 3 confirm.

1. Dependence of the real part of the complex angle of refraction on the angle of incidence at different degrees of soil wetting.

2. Dependence of the reflection coefficient on the angle of incidence and the parameters of the first and second soil layer - with an increase in different degrees of soil wetting, the minimum of function (Brewster point) shifts to the right.

Thus, the reflected and refracted signal contains sufficient information on the dielectric properties of the soil layer under study.

Fig. 4 shows the results of modeling the reflected signal when using a bistatic radar system for soil moisture research.

Analysis of the simulation results (see Fig. 4) shows that the use of a bistatic radar system with irradiation of the earth's surface at an angle close to the value of Brewster's angle provides better conditions for observing the signal reflected from the soil layer, which is located above the groundwater level.

The task of determining the moisture content of subsurface soil layers can be decomposed into the following particular tasks. 
1. Picking the components out of the resultant received signal that are formed from the soil layers of various depths, up to the groundwater level.

2. Detection of the amplitude and phase of the signal received from a given soil layer.

3. Assessment of the dielectric constant of the soil layer and determining the moisture percentage.
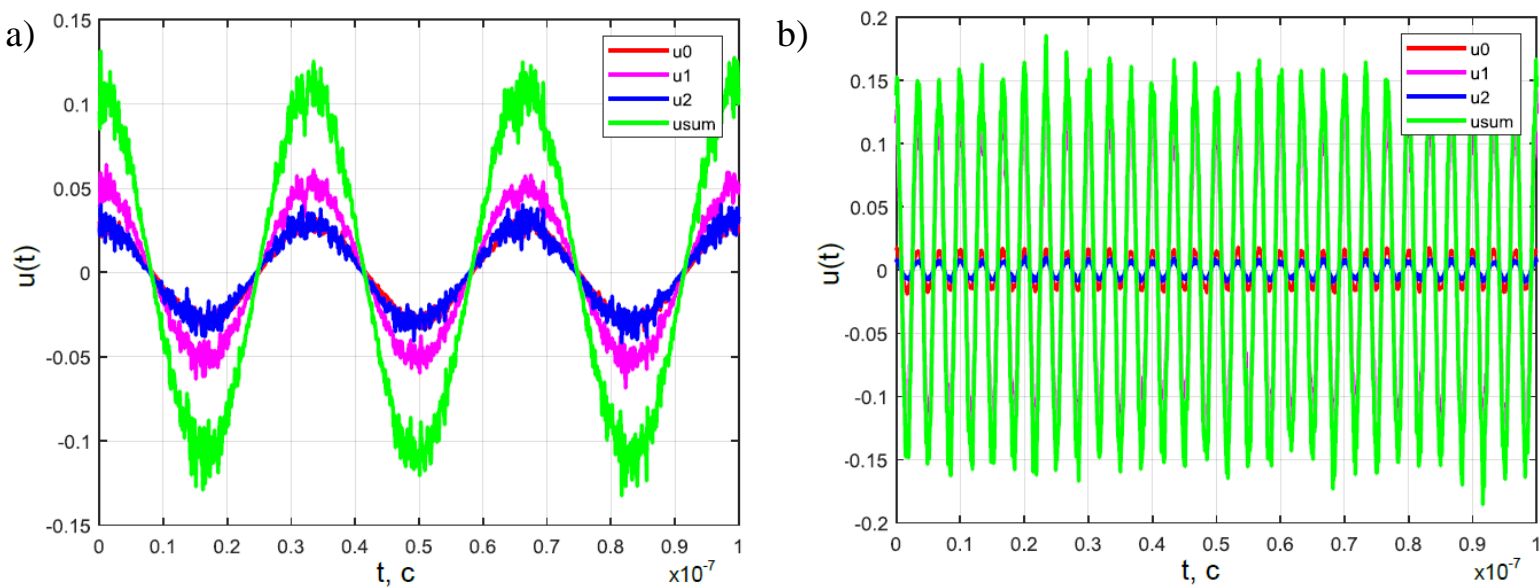

Fig. 4. Results of modeling the process of the reflected signal formation when using a bistatic radar system and sounding the soil at Brewster angle equal to 58 degrees: a - frequency of the sounding signal is $30 \mathrm{MHz}$; - frequency of the probing signal is $300 \mathrm{MHz}$

A correlation detector can be used to pick the components of the radar response from different soil layers.

Solving the inverse problem - calculating the correlation coefficient for a given value of the phase shift (distance from the earth's surface), one can determine the level of the signal coming from this layer. Taking into account the known dependence of the signal level and reflection coefficient on the dielectric constant and specific conductivity of soil, as well as the dependence of the dielectric constant on soil moisture, the moisture percentage in the soil layers is determined.

Fig. 5a shows the results of modeling a $27 \mathrm{MHz}$ radar signal reflected from different layers; Fig. 5b shows the calculated correlation coefficients for different soil layers.
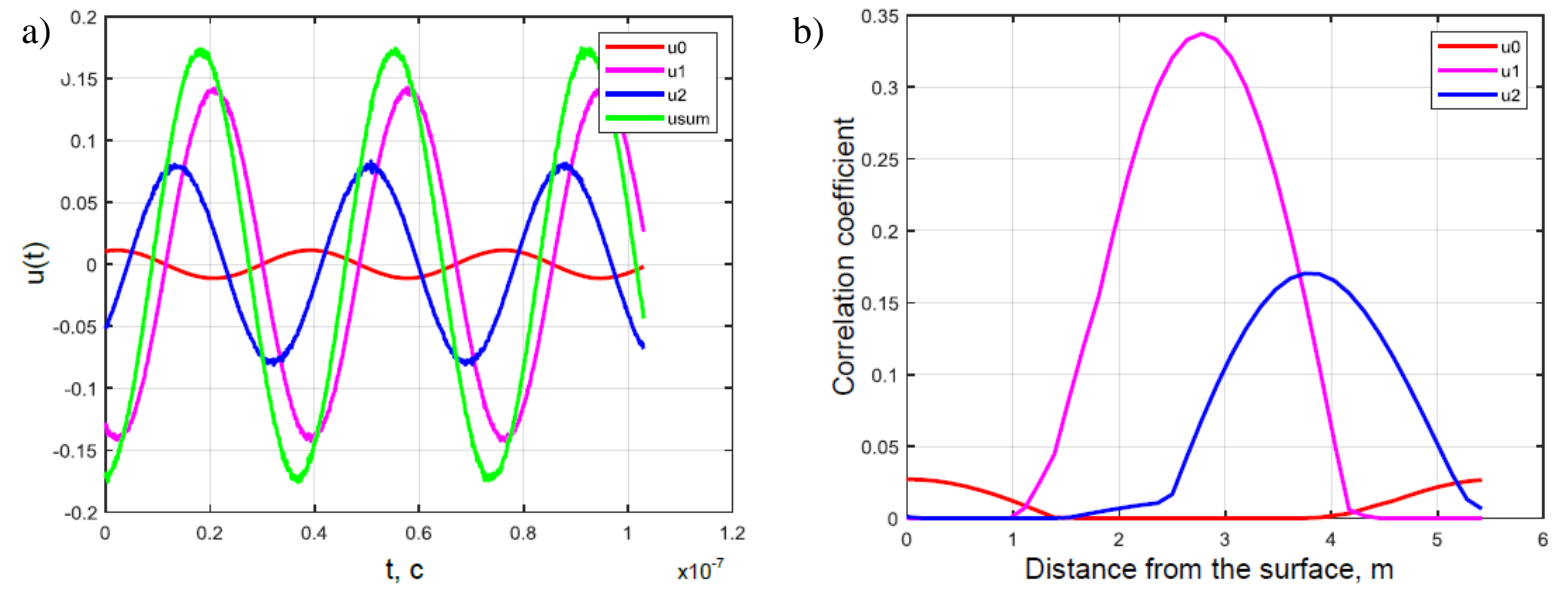

Fig. 5. Detection of the reflected signal from a given soil layer in the received signal

As follows from the analysis of the simulation results (see Fig. 5a), the radar signal reflected from different soil layers has a different phase shift value - with an increase in the interface depth of soil layers with different properties, the phase shift value changes. By the maximum of the correlation coefficient (see Fig. 5b) the depth of the soil layer with individual properties can be clearly judged.

The distance from the ground surface to the depth is determined relative to the signal reflected from the air-surface boundary. The phase delay of the signal reflected from the air-surface interface can be calculated based on the distance travelled by the electromagnetic wave radiated from a height $H$ at an angle close to Brewster's angle $\varphi_{B}$ : 


$$
L=\frac{H}{\cos \left(\varphi_{B}\right)}+\sqrt{H^{2}+\left(d-\frac{H \sin \left(\varphi_{B}\right)}{\sin \left(0.5 \pi-\varphi_{B}\right)}\right)},
$$

where $d=\sqrt{\left(x_{2}-x_{1}\right)^{2}+\left(y_{2}-y_{1}\right)^{2}+\left(z_{2}-z_{1}\right)^{2}}, x_{2}, x_{1}, y_{2}, y_{1}, z_{2}, z_{1}-$ coordinates of the transmitting and receiving positions of the bistatic system.

To determine the coordinates of the bistatic radar system, the equipment of the satellite navigation system can be used.

The correlation detector is located in the receiving equipment. The range between the UAVs is calculated according to the information about the coordinates of transmitting position, obtained using a radio modem.

Research in the field of radar sensing of soils is limited mainly to determining the properties of the soil surface, without penetrating into deep layers [1;3;6; 7]. Similar studies [14] led to similar results: a correlation was found between the backscattering coefficients of vertically polarized radars and most of these properties, the magnitude and direction of the correlation for most of the soil properties are markedly different.

\section{Conclusions}

The method of soil moisture analysis by a radar bistatic system with irradiation of the earth's surface at Brewster angle was examined using mathematical modeling. The following conclusions can be drawn from the study.

1. Observation of the radar signal reflected from subsurface soil layers up to the groundwater level occurs with less influence of the signal reflected from the air-soil boundary;

2. The amplitude of the reflected signal depends on the dielectric constant of the soil and, consequently, on its moisture;

3. To tie the signal amplitude to the distance from the "air-soil" boundary, the phase method can be used. In this case, to minimize ambiguous distance measurements, it is necessary to use a radio signal with a wavelength exceeding the desired depth of the studied soil layer;

4. Numerical moisture characteristics for different soils should be calibrated using experimental studies.

\section{References}

[1] Baghdadi N., Zribi M. Characterization of Soil Surface Properties Using Radar Remote Sensing, Land Surface Remote Sensing in Continental Hydrology. Elsevier, 2016. 458p.

[2] Han D., Vahedifard F., Aanstoos J.V. Investigating the correlation between radar backscatter and in situ soil property measurements.International Journal of Applied Earth Observation and Geoinformation, Vol. 95, 2003,pp.136-144.

[3] Bonadies S., Gadsden S.A. An overview of autonomous crop row navigation strategies for unmanned ground vehicles. Engineering in Agriculture, Environment and Food, Vol.12, Issue 1, 2019, pp. 24-31.

[4] Fu H., Liu Z., Guo X., Cui H. Double-frequency ground penetrating radar for measurement of ice thickness and water depth in rivers and canals: Development, verification and application. Cold Regions Science and Technology, Vol. 154, 2018, pp. 85-94.

[5] Wu K., Rodriguez G.A., Zajc M. etc. A new drone-borne GPR for soil moisture mapping, Remote Sensing of Environment, Vol. 235, 2019, 111456

[6] Petropoulos G.P., Ireland G., Barrett B. Surface soil moisture retrievals from remote sensing: Current status, products \& future trends. Physics and Chemistry of the Earth, Vol. 83-84, 2015, pp.36-56.

[7] Grunwald S., Vasques G.M., Rivero R.G. Fusion of Soil and Remote Sensing Data to Model Soil Properties. Advances in Agronomy, Academic Press, Vol.131, 2015, pp.1-109.

[8] Thirion-Lefevre L., Guinvarc'h R.The double Brewster angle effect. Comptes Rendus Physique, Vol. 19, 2018, pp. 43-53.

[9] Mohamed A.-M.O., Evan K. Paleologos L.K. Dielectric Permittivity and Moisture Content. Fundamentals of Geoenvironmental Engineering, Butterworth-Heinemann, 2018, pp. 581-637 
[10] Lekshmi S.U., Singh D.N., Baghini M.S. Investigations on magnetic characteristics of the soil and their influence on its dielectric response. Applied Clay Science, Vol. 158, 2018, pp.113-122.

[11]Калинкевич А.А. и др. Многочастотная радиолокация “эффективной” влажности почвы (Multifrequency radiolocation of "effective" soil moisture). RENSIT, 2018, 10(2):193-208. DOI: 10.17725/rensit.2018.10.19 (in Russian).

[12]Баженов А.В. Электродинамика и распространение радиоволн: Учебное пособие (Electrodynamics and Radio Wave Propagation: Textbook). Stavropol: STIS, 2011. - 244 p., ill. (In Russian).

[13]Боярский Д.А., Тихонов В.В. Учет диэлектрических свойств связанной воды при моделировании эффективной диэлектрической проницаемости влажных почв в СВЧ диапазоне (Taking into account the dielectric properties of bound water in modeling the effective dielectric constant of moist soils in the microwave range). Radio engineering and electronics, 1998. Vol. 43. No 4, pp. 446-454. (In Russian).

[14] Han D., Vahedifard F., Aanstoos J.V. etc. Investigating the correlation between radar backscatter and in situ soil property measurements, International Journal of Applied Earth Observation and Geoinformation, Vol. 57, 2017, pp. 136-144. 